\title{
The effect of "early"protein of papillomavirus HPV16 E2 made in plant expression system on the base of tomato fruit on tumor formation in mice infected with cancer HeLa cells
}

\author{
Rekoslavskaya N.I.*, Salyaev R.K., Stolbikov A.S. \\ The Siberian institute of plant physiology and biochemistry of the Siberian branch of Russia Academy \\ of Sciences, Irkutsk, Russia \\ * email: rekoslavskaya@sifibr.irk.ru
}

The plant expression system was developed for purposes of the synthesis of the heterologous protein HPV16 E2 (and others) in tomato fruits. The sequence of the RNA replicase of the simple plant retrovirus (Cucumber mosaic virus) RdRP CMV (the gene RNA 2a) was inserted into genetic construct which contained the sequence of the antisilencer protein encoded by conjugated gene RNA $2 b$ either. As a whole, the construct was presented as followed: $R B$->p35S RdRP $(2 a+2 b)$->p35S hpv16 E2 t35S$>L B$, in which both promoters $\mathrm{p} 35 \mathrm{~S}$ were placed as two nonhomologous sequences. The synthetic yield of the protein of interest HPV16 E2 was as high as 25-30\% of the total soluble protein (up to $300 \mathrm{mkg}$ per $1 \mathrm{mg}$ of TSP). This amount of HPV16 E2 synthesized allowed to use transgenic tomato fruit as vaccine material for mice vaccination without any preliminary purification. To rise antibodies in mice blood serum, mice were vaccinated per os with vaccine material of tomato fruit transgenic with the gene hpvl6 E2 for 3-4 times (in different experiments) and after one month from last vaccination, samples of blood were collected and used for the analysis of antibodies in serum. To evaluate the aftereffect of the vaccination, the rest part of vaccinated mice have been stayed alive for sufficiently long time (more than one year). It was found that vaccinated mice long lived and were very active (there were almost no deaths). The content and quality of antibodies to HPV16 E2 from blood serum of vaccinated mice were analysed by Elisa and Western blot hybridisation. It was shown that antibodies raised to HPV16 E2 in blood of vaccinated mice have higher titer and significantly prominent avidity in comparison to commercial antibodies of the Abcam company (UK) developed to E2vaccinia conjugated with the protein binding of maltose after intravenous administration to mice. The HPV16 E2 protein is the main regulatory protein of the expression, replication and releasing in the live cycle of papillomavirus. The gene encoded HPV16 E2 is placed within the promoter of "early" genes $h p v$ E6 and $h p v$ E7 encoded oncogenes HPV E6 and HPV E7 and played the role of the supersupressor of the expression of these genes. HPV16 E2 is absent in tumors because of the degradation during the integration of papillomavirus. Therefore it was very significant to appreciate the action of this protein on tumors appeared in mice after the injection of cancer HeLa cells in hips. Conventional laboratory mice (male and female at the age of 6 months) were infected with $100 \mathrm{mkl}$ of the suspension of HeLa cells into hips. After $1 \mathrm{month}$, the appearance of lung tumors in both females and males as well testis tumors in males were observed. Oral administration of vaccine material with HPV16 E2 in transgenic tomato fruit to mice caused the regression of tumors both in lungs of males and females and the siginificant decrease of the size of tumors of testis in males. The regression of tumors was accompanied by the increase of contents of $\mathrm{T}$ cell receptor, interferon, CD8+ and CD4+ T lymphocytes as well apoptotic enzymes: granzyme B, perforin and granulysin in blood serum and splenocytes according to Elispot analysis. 